\title{
Kriging and Latin Hypercube Sampling assisted simulation optimization in optimal design of PID controller for speed control of DC motor
}

\begin{abstract}
Investigating less computationally expensive methods for achieving optimal tuning of proportional, integral, and derivative gains in PID controller also has become a main challenging topic in the world of control systems. This paper aims to propose a new lessexpensive method to the optimal design of PID controller. For this purpose, Kriging metamodel is used with Latin Hypercube Sampling (LHS) as a common experimental design method in the class of space filling design. Kriging can interpolate over whole design space and assisted to investigate global optimum point. A numerical case in the tuning of PID controller for linear speed control of DC motor is served to show the applicability and superiority of proposed method compared to two existing methods such as traditional ZeiglerNichols method and Taguchi-Grey Relational Analysis (Taguchi-GRA).
\end{abstract}

Keyword: Computer experiments; DC motor; Kriging; Latin Hypercube Sampling; Metamodel; PID controller; Simulation optimization 